\title{
Modal and Probabilistic Analysis of Wind Turbine Blade under Air-Flow
}

\author{
Ismaïl Sossey-Alaoui*, Bouchaïb Radi ** \\ *(Laboratoire Ingénierie Management Industriel et Innovation, FST-Settat, Hassan 1st University, Morocco. \\ **(Department of Technic, Laboratoire Ingénierie Management Industriel et Innovation, Hassan 1st \\ University, Morocco
}

\begin{abstract}
Wind power is one of the most important sources of renewable energy. Wind-turbines extract kinetic energy from the wind and convert it into mechanical energy. Therefore wind turbine power production depends on the interaction between the blade and the wind. The fluid-structure interaction, that means the interaction of some deformable structure with a surrounding or internal fluid flow, belong nowadays to the most important and challenging multi-physics problems which are aimed to treat by numerical simulations. The topic fluid-structure interaction plays a dominant role in many fields of engineering. Therefore, a strong need for appropriate numerical simulation tools exists with a variety of numerical and physical aspects. The present paper takes care about fluid-structure interaction using modern simulation techniques such as coupled field analysis. This work illustrates the use of load transfer coupled physics analysis to solve a steady-state air flow-blade interaction problem, followed by modal analysis where natural frequency are obtained with two different approaches: deterministic and probabilistic. The numerical results are deduced from a finite element approximation of the coupled problem with a non-symmetric pressure/displacement formulation. Determin istic and probabilistic results are given and discussed.
\end{abstract}

Keywords: Fluid structure interaction, muti-physics problems, aerodynamic, modal analysis, Monte Carlo, Win turbine blade.

\section{INTRODUCTION}

Countries around the world are putting substantial effort into the development of wind energy technologies. The ambitious wind energy goals put pressure on the wind energy industry research and development to significantly enhance current wind generation capabilities in a short period of time and decrease the associated costs [1].

A wind turbine is a device that extracts kinetic energy from the wind and converts it into mechanical energy. Therefore wind turbine power production depends on the interaction between the blade and the wind [2, 3].

Fluid-structure interaction (FSI) is a class of problems with mutual dependence between the fluid and structural mechanics parts. The flow behavior depends on the shape of the structure and its motion, and the motion and deformation of the structure depend on the fluid mechanics forces acting on the structure. We see FSI almost everywhere in engineering, sciences, and medicine, and also in our daily lives [1].

In engineering applications, FSI plays an important role and influences the decisions that go into the design of systems of contemporary interest. Therefore, truly predictive FSI methods, which help address these problems of interest, are in high demand in industry, research laboratories, medical fields, space exploration, and many other contexts.
We see some use of analytical methods in solution of fluid-only or structure-only problems, there are very few such developments in solution of FSI problems. In contrast, there have been significant advances in computational FSI research, especially in recent decades, in both core FSI methods forming a general framework and special FSI methods targeting specific classes of problems.

Finite Element Analysis (FEA) is a numerical simulation method that can be used to calculate the response of a complicated structure due to the application of forcing functions, which could be used to demonstrate the nonlinear largedeflection structural coupling for a fluid domain. This method is a powerful computational technique for approximate solutions to a variety of "realworld" engineering problems having complex domains subjected to general boundary conditions. FEA has become an essential step in the design or modeling of a physical phenomenon in various engineering disciplines. A physical phenomenon usually occurs in a continuum of matter (solid, liquid, or gas) involving several field variables. The field variables vary from point to point, thus possessing an infinite number of solutions in the domain [4].

A coupled-field analysis is a combination of analyses from different engineering disciplines (physics fields) that interact to solve a global 
engineering problem. When the input of one field analysis depends on the results from another analysis, the analyses are coupled [5]. This technique is useful for solving problems where the coupled interaction of phenomena from various disciplines of physical science is significant.

A modal analysis method avoids moving to the formal calculation that requires huge calculations, and allows for a simple and fast against rough calculation of own frequencies. Because the structural frequencies are not known a priori, the finite element equilibrium equations for this type of analysis involve the solution of homogeneous algebraic equations whose eigenvalues correspond to the frequencies, and the eigenvectors represent the vibration modes [4].

Taking account in to uncertainties in the mechanical analysis it is necessary for optimal and robust structures design. Indeed, it is widely recognized that small uncertainties in the system settings can have a considerable influence on its vibratory behavior expected [6].

The present work illustrates in the first way a steady-state fluid-structure interaction problem applied on a blade wind turbine subject to air flow, this problem demonstrates the use of nonlinear large-deflection structural coupling for a fluid domain; Followed by modal analysis to calculate the natural frequencies and mode shapes of blade subjected to air flow. In the second way we used probabilistic approach to improve the robustness of the design and estimate the impact of uncertainties of parameters on the vibration response of the blade.

\section{COUPLED FIELD ANALYSIS}

A coupled-field analysis is an analysis that takes into account the interaction (coupling) between two or more disciplines (fields) of engineering; hence, we often refer to a coupled-field analysis as a multi-physics analysis.

Some cases use only one-way coupling. For example, the calculation of the flow field over a cement wall. More complicated cases involve twoway coupling. In a fluid-structure interaction problem, the fluid pressure causes the structure to deform, which in turn causes the fluid solution to change. This problem requires iterations between the two physics fields for convergence [5]. The coupling between the fields can be accomplished by either direct or load transfer coupling. Coupling across fields can be complicated because different fields may be solving for different types of analyses during a simulation. The term load transfer coupled physics refers to using the results of one physics simulation as loads for the next. If the analyses are fully coupled, results of the second analysis will change some input to the first analysis.
We perform load transfer coupling analysis, with a nonlinear transient fluid-solid interaction analysis, using FLOTRAN and ANSYS structural coupled field elements.

\section{GOVERNING EQUATIONS}

In this part, we present the partial differential equations that govern the fluid and structural mechanics parts of the fluid-structure interaction (FSI) problem. The fluid and structural mechanics equations are complemented by the applicable boundary conditions and constitutive models [1].

\subsection{Fluid solver}

The fluid mechanics part of the FSI problem is governed by the Navier-Stokes equations of incompressible flows.

Let $\Omega_{t} \in I R^{n} \quad(\mathrm{n}=2,3)$, be the spatial fluid mechanics domain with boundary $\Gamma_{t}$ at time $\mathrm{t} \in(0, \mathrm{~T})$. The subscript $\mathrm{t}$ indicates that the fluid mechanics spatial domain is time-dependent. The Navier-Stokes equations of incompressible flows may be written on $\Omega_{t}$ and $\forall \mathrm{t} \in(0, \mathrm{~T})$ as

$$
\left\{\begin{array}{c}
\rho \frac{\partial u^{f}}{\partial t}+\rho\left(u^{f} . \nabla\right)-u^{f}-\nabla \cdot \sigma\left(u^{f}, p\right)=\rho_{f} \\
\nabla \cdot u^{f}=0
\end{array}\right.
$$

where $\rho, u$, and $f$ are the density, velocity, and the external force (per unit mass), respectively, and the stress tens or $\sigma$ is defined as

$$
\sigma\left(u^{f}, p\right)=-p I+2 \mu \varepsilon\left(u^{f}\right)
$$

Here $\mathrm{p}$ is the pressure, $\mathrm{I}$ is the identity tensor, $\mathrm{u}$ is the dynamic viscosity, and $\Sigma(\mathrm{u})$ is the strain-rate tensor given by

$$
\varepsilon\left(u^{f}\right)=\frac{1}{2}\left(\nabla u^{f}+\left(\nabla u^{f}\right)^{T}\right)
$$

Equation (1) represents the local balance of linear momentum and mass. The momentum balance equation is written in the so-called conservative form.

For incompressible flows, we can write the momentu m equation also as

$\rho\left(\frac{\partial u^{f}}{\partial t}+\nabla \cdot\left(u^{f} \otimes u^{f}\right)-\mathrm{f}\right)-\nabla \cdot \sigma=0$

For constant density, Equation (4) represents the conservative form of the mo mentum equation.

$$
\begin{gathered}
\rho\left(\frac{\partial u^{f}}{\partial t}+u^{f} \cdot \nabla u^{f}-\mathrm{f}\right)-\nabla \cdot \sigma=0 \\
\nabla \cdot u^{f}=0
\end{gathered}
$$

Assuming a fixed Cartesian basis on $\mathrm{IR}^{3}$, we let indices $i$ and $j$ take on the values 1,2,3. We let $u_{i}$ denote the $i^{t h}$ Cartesian component of $\mathbf{u}$, and let $x_{i}$ 
denote the $i^{\text {th }}$ component of $\mathbf{x}$. We denote differentiation by a comma (e.g., $\left.u_{i, j}=u_{i, x j}=\partial u_{i} / \partial x_{j}\right)$. We will also use the summation convention, in which repeated indices imply summation; e.g., in $\mathrm{IR}^{3}$,

$u_{i, j j}^{f}=u_{i, 11}^{f}+u_{i, 22}^{f}+u_{i, 33}^{f}=\frac{\partial^{2} u_{i}^{f}}{\partial x_{1}^{2}}+\frac{\partial^{2} u_{i}^{f}}{\partial x_{2}^{2}}+\frac{\partial^{2} u_{i}^{f}}{\partial x_{3}^{2}}(6)$

So the Navier-Stokes equations of incompressible flows can be rewritten as:

$$
\begin{aligned}
\rho\left(u_{i, t}^{f}+u_{j}^{f} u_{i, j}^{f}-\mathrm{f}_{i}\right)-\sigma_{i j, j} & =0 \\
u_{i, i}^{f} & =0
\end{aligned}
$$

where

$$
\begin{gathered}
\sigma_{i j}=-p \delta_{i j}+2 \mu \varepsilon_{i j} \\
\varepsilon_{i j}=\frac{1}{2}\left(u_{i, j}^{f}+u_{j, i}^{f}\right)
\end{gathered}
$$

and $\delta i j$ is the Kronecker delta.

In general, on a given part of the spatial boundary, either kinematic or traction boundary conditions are prescribed. Kinematic boundary conditions are also referred to as essential or Dirichlet, while traction boundary conditions are also called natural or Neumann. The essential and natural boundary conditions for Equation (6) are

$$
\begin{gathered}
u_{i}^{f}=g_{i} \text { on }\left(\Gamma_{t}\right)_{g i} \\
\sigma_{i j} n_{j}=h_{i} \text { on }\left(\Gamma_{t}\right)_{h i}
\end{gathered}
$$

where, for every velocity component i, $(\Gamma \mathrm{t}) \mathrm{gi}$ and $(\Gamma \mathrm{t}) \mathrm{hi}$ are the complementary subsets of the domain boundary $\Gamma \mathrm{t}$, ni's are components of the unit outward normal vector $\mathrm{n}$, and gi and hi are given functions.

The Stokes equations are obtained by neglecting the convective terms in Equation (5), that is,

$$
\left\{\begin{aligned}
\rho\left(\frac{\partial u^{f}}{\partial t}-\mathrm{f}\right)-\nabla \cdot \sigma & =0 \\
\nabla \cdot u^{f} & =0
\end{aligned}\right.
$$

The above model is used for describing very slow (e.g., "creeping") flows. Note that the Stokes equations are linear with respect to both velocity and pressure, while the Navier-Stokes equations are not.

The other special case corresponds to inviscid flows described by the Euler equations of incompressible flows, namely

$$
\left\{\begin{array}{r}
\rho\left(\frac{\partial u^{f}}{\partial t}+u^{f} \cdot \nabla u^{f}-\mathrm{f}\right)-\nabla p=0 \\
\nabla \cdot u^{f}=0
\end{array}\right.
$$

The Eu ler equations retain the quadratic nonlinearity of the convective term.

The variational formulation can now be expressed as: Find $\left(\mathrm{u}_{\mathrm{f}}, \mathrm{p}\right) \in \mathrm{U} \times \mathrm{Q}$ such that

$\left(\rho \frac{\partial u^{f}}{\partial t}, v\right)+c\left(u^{f} ; u^{f}, v\right)+b(p, v)+a\left(u^{f}, u^{f}\right)+b\left(q, u^{f}\right)=f(v)(13)$

For $(v, q) \in V \times Q$. We have defined the spaces

$U=H_{\Gamma_{D}, \Gamma_{M}^{\perp}}(\Omega)=\left\{v \in H^{1}(\Omega) \mid v=u_{D}^{f}\right.$ on $\Gamma_{D}$ et $v \cdot n=u_{\perp}^{f}$ on $\left.\Gamma_{M}\right\}$

$V=H_{\Gamma_{D}, \Gamma_{M}^{\perp}}(\Omega)=\left\{v \in H^{1}(\Omega) \mid v=0\right.$ on $\Gamma_{D}$ et $v \cdot n=0$ on $\left.\Gamma_{M}\right\}$

$Q=L^{2}(\Omega)$,

where $u_{D}^{f}$ and $u_{\perp}^{f}$ both are given functions and $\boldsymbol{n}$ is the unit outer normal on $\Gamma$. We have also defined the forms

$$
\begin{aligned}
& a(u, v)=2 \int_{\Omega} \mu \varepsilon(u): \varepsilon(v) d x \\
& b(q, v)=-\int_{\Omega} \mu(\nabla . v) q d x \\
& c(w, u, v)=\int_{\Omega} \rho(w \cdot \nabla) u \cdot v d x \\
& f(v)=\int_{\Omega} \rho f . v d x+\int_{\Gamma_{N}} t . v d s
\end{aligned}
$$

where $\mathrm{t}=\sigma \cdot \mathrm{n}$ is the traction vector on $\Gamma$.

\subsection{Structural Sol ver}

Let $\Omega_{0} \in I R^{n}$ be the material do main of a structure in the reference configuration, and let $\Gamma_{0}$ be its boundary. Let $\Omega_{t} \in I R^{n}, \mathrm{t} \in(0, \mathrm{~T})$, be the material domain of a structure in the current configuration, and let $\Gamma_{\mathrm{t}}$ be its boundary.

The velocity $u$ and acceleration a of the structure are obtained by differentiating the displacement $\mathrm{y}$ with respect to time holding the material coordinate $\mathrm{X}$ fixed, namely

$u=\frac{d y}{d t}$ and $a=\frac{d^{2} y}{d t^{2}}$

The deformation gradient $\mathbf{F}$ is given by

$F=\frac{\partial x}{\partial X}=I+\frac{\partial y}{\partial X}$

which we use to define the Cauchy-Green deformation tens or $\mathbf{C}$ as

$\mathrm{C}=\mathrm{F}^{\mathrm{T}} \mathrm{F}$ (17)

and the Green-Lagrange strain tensor $\mathbf{E}$ as

$\mathrm{E}=\frac{1}{2}(C-I)$

and $\quad J=\operatorname{det} \mathbf{F}$. (19)

The Cartesian components of the deformation gradient is given bay

$$
F_{i I}=\frac{\partial x_{i}}{\partial X_{I}}=\delta_{i I}+\frac{\partial y_{i}}{\partial X_{I}}
$$

- Variational Formu lation 
The principle of virtual work

$$
\delta W=\delta W_{\text {int }}+\delta W_{\text {ext }}=0
$$

where $\mathrm{W}, \mathrm{Wint}$, and Wext are the total, internal, and external work, respectively, and $\delta$ denotes their variation with respect to the virtual displacement $\mathrm{w}$. Here $\delta$ Wext includes the virtual work done by the inertial and body forces and surface tractions, and is given by

$$
\delta W_{\text {ext }}=\int_{\Omega_{t}} \mathrm{~W} \cdot \rho(f-a) d \Omega+\int_{\left(\Gamma_{t}\right)_{h}} \text { w.h d } \Gamma
$$

The expression for the internal virtual work for a composite shell may be compactly written as

$$
\begin{array}{r}
\delta W_{\text {int }}=-\int_{\Gamma_{0}^{s}} \delta \bar{\varepsilon} \cdot\left(K_{\text {exte }} \bar{\varepsilon}+K_{\text {coup }} \bar{\kappa}\right) d \Gamma- \\
\int_{\Gamma_{0}^{s}} \delta \bar{\kappa} \cdot\left(K_{\text {coup }} \bar{\varepsilon}+K_{\text {bend }} \bar{\kappa}\right) d \Gamma
\end{array}
$$

We employ the composite Kirchhoff-Love shell formulation to model the structural mechanics of wind-turbine blades. Composite materials are typically used in the manufacturing of modern windturbine blades.

The complete variational formulation of the Kirchhoff-Love shell is given by: find the shell midsurface displacement $y^{h} \in S_{y}^{h}$, such that

$$
\begin{aligned}
& \forall \mathrm{w}^{h} \in v_{y}^{h}: \\
& \int_{\Gamma_{0}^{s}} \mathrm{~W}_{2}^{h} \cdot \bar{\rho}_{0} \mathrm{~h}_{\mathrm{th}}\left(\frac{d^{2} y^{h}}{d t^{2}}-\mathrm{f}^{h}\right) \mathrm{d} \Gamma \\
& +\int_{\Gamma_{0}^{s}} \delta \bar{\varepsilon}^{h} \cdot\left(\mathrm{K}_{\text {exte }} \bar{\varepsilon}^{h}+\mathrm{K}_{\text {coup }} \bar{\kappa}^{h}\right) \mathrm{d} \Gamma \\
& +\int_{\Gamma_{0}^{s}} \delta \bar{\kappa}^{h} \cdot\left(\mathrm{K}_{\text {coup }} \bar{\varepsilon}^{h}+\mathrm{K}_{\text {bend }} \bar{\kappa}^{h}\right) \mathrm{d} \Gamma \\
& +\int_{\Gamma_{0}^{b}} \delta \bar{\kappa}^{h} \cdot \mathrm{K}_{\text {bstr }} \bar{\kappa}^{h} \mathrm{~d} \Gamma-\int_{\left(\Gamma_{t}^{s}\right)_{h}} \mathrm{w}_{2}^{h} \cdot \mathrm{h}^{h} \mathrm{~d} \Gamma=0
\end{aligned}
$$

In the above formulation, the superscript $\mathrm{h}$ denotes the discrete nature of the quantities involved. Here Kbstr is the bending stiffness of the strips:

$$
K_{b s t r}=\frac{h_{t h}^{3}}{12} \bar{C}_{b s t r} \text { (25) }
$$

where

$$
\bar{C}_{b s t r}=\left[\begin{array}{ccc}
E_{s} & 0 & 0 \\
0 & 0 & 0 \\
0 & 0 & 0
\end{array}\right]
$$

and $E_{S}$ is the scalar bending-strip stiffness, typically chosen as a multiple of the local Young's modulus of the shell. The stiffness $\mathrm{E}_{\mathrm{s}}$ must be high enough so that the change in angle is within an acceptable tolerance. However, if $\mathrm{E}_{\mathrm{s}}$ is chosen too high, the global stiffness matrix becomes badly conditioned, which may lead to divergence in the computations.
And $\left(\Gamma_{t}^{s}\right)_{h}$ is the shell subdomain with a prescribed traction boundary condition, and $\bar{\rho}_{0}$ is the throughthickness-averaged shell density given by

$\bar{\rho}_{0}=\frac{1}{h_{t h}} \int_{h_{t h}} \rho_{0} \mathrm{~d} \xi_{3}$

Here $\mathrm{S} y$ and $V y$ are the sets of trial and test functions for the structural mechanics problem, defined as

$S_{y}=\left\{y \mid y(., t) \in\left(H^{1}\left(\Omega_{t}\right)\right)^{n}, y_{i}=g_{i}\right.$ on $\left.\left(\Gamma_{t}\right)_{g i}\right\}(28)$ and

$v_{y}=\left\{\mathrm{W} \mid \mathrm{w}(., t) \in\left(H^{1}\left(\Omega_{t}\right)\right)^{n}, \mathrm{w}_{i}=0\right.$ on $\left.\left(\Gamma_{t}\right)_{g i}\right\}(29)$

Here, for each $i, \quad(\Gamma t)_{\mathrm{g} i}$ and $(\Gamma t)_{\mathrm{h} i}$ are the complementary subsets of the domain boundary $\Gamma t$, and $g i$ is a given function.

Pre-integrating through the shell thickness in Equation (25), the extensional stiffness $\mathbf{K}_{\text {exte, }}$, coupling stiffness $\mathbf{K}_{\text {coup }}$, and bending stiffness $\mathbf{K}_{\text {bend }}$ are given by

$$
\begin{aligned}
& \kappa_{\text {exte }}=\int_{h_{t h}} \overline{\mathrm{C}} \mathrm{d} \xi_{3}=\frac{h_{t h}}{n} \sum_{k=1}^{n} \overline{\mathrm{C}}_{k}, \text { (30) } \\
& \kappa_{\text {coup }}=\int_{h_{t h}} \xi_{3} \overline{\mathrm{C}} \mathrm{d} \xi_{3}=\frac{h^{2}}{n^{2}} \sum_{k=1}^{n} \overline{\mathrm{C}}_{k}\left(k-\frac{n}{2}-\frac{1}{2}\right)(31) \\
& \kappa_{\text {bend }}=\int_{h_{t h}} \xi_{3}^{2} \overline{\mathrm{C}} \mathrm{d} \xi_{3}=\frac{h_{\text {th }}^{3}}{n^{3}} \sum_{k=1}^{n} \overline{\mathrm{C}}_{k}\left(\left(k-\frac{n}{2}-\frac{1}{2}\right)^{2}+\frac{1}{12}\right)(32)
\end{aligned}
$$

where

$\overline{\mathrm{C}}_{k}=T^{T}\left(\phi_{k}\right) \overline{\mathrm{C}}_{\text {ort }} T\left(\phi_{k}\right)$

$T(\phi)=\left[\begin{array}{ccc}\cos ^{2} \phi & \sin ^{2} \phi & \sin \phi \cos \phi \\ \sin ^{2} \phi & \cos ^{2} \phi & -\sin \phi \cos \phi \\ -2 \sin \phi \cos \phi & 2 \sin \phi \cos \phi & \cos ^{2} \phi-\sin ^{2} \phi\end{array}\right]$

\subsection{Solver Coupling}

We couple the fluid and the structural solver at the interface using a Dirichlet-Neumann coupling. The coupling conditions are

$$
\left\{\begin{array}{c}
v_{\Gamma}^{f}=\frac{d u_{\Gamma}}{d t} \\
\sigma_{\Gamma}^{f} \cdot n=\sigma_{\Gamma}^{s} \cdot n
\end{array}\right.
$$

where $\boldsymbol{n}$ is the unit normal vector to the interface $\Gamma$.

\section{NUMERICAL COUPLING SIMULATION}

The ANSYS program performs load transfer coupled physics analyses using the concept of a physics environment. The term physics environment applies to both a file you create which 
contains all operating parameters and characteristics for a particular physics analysis and to the file's contents.

In the ANSYS program, we can perform a load transfer coupled-field analysis using either separate databases or a single database with multiple physics environments. We use a single database and multiple physics environments. In this approach, a single database must contain the elements and nodes for all the physics analyses that you undertake, and allows us to quickly switch between physics environments, which is ideally suited for fully coupled scenarios requiring multiple passes between physics solutions.

The object of this work is to determine the pressure drop and blade deflection under steadystate conditions. The blade will deform due to the fluid pressure. The deflection may be significant enough to affect the flow field. By solving a structural analysis in the structural region, we obtain the blade displacements that you need to morph the region around the blade. We then use the morphed mesh in a subsequent fluid analysis. The fluid analysis uses null type elements for the blade and the structural analysis uses null type elements for the fluid.

\subsection{Mash di vision and boundary conditions}

Many times a coupled-field analysis involving a field domain (fluid) and a structural domain yields significant structural deflections. In this case, to obtain an overall converged coupledfield solution it is often necessary to update the finite element mesh in the non-structural region to coincide with the structural deflection and recursively cycle between the field solution and structural solution.

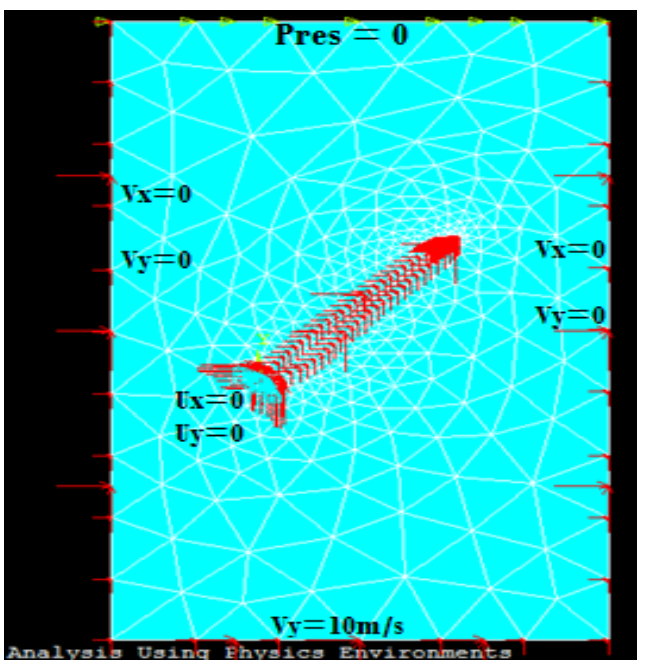

Fig.1: Area of two physics domains

\subsection{Results}

The fluid-structure solution loop was executed until the convergence criteria were met. A convergence tolerance of $0.5 \%$ was used. For the first analysis, 50 global iterations were sufficient to converge the FLOTRAN solution. In the Fluid Structure interaction loop, the number of iterations was set to 50 for the remaining FLOTRAN runs.

Figure 2: Streamlines near Blade, depicts the streamlines near the blade for the deformed geometry and Figure 3: Pressure Contours, the pressure contours.

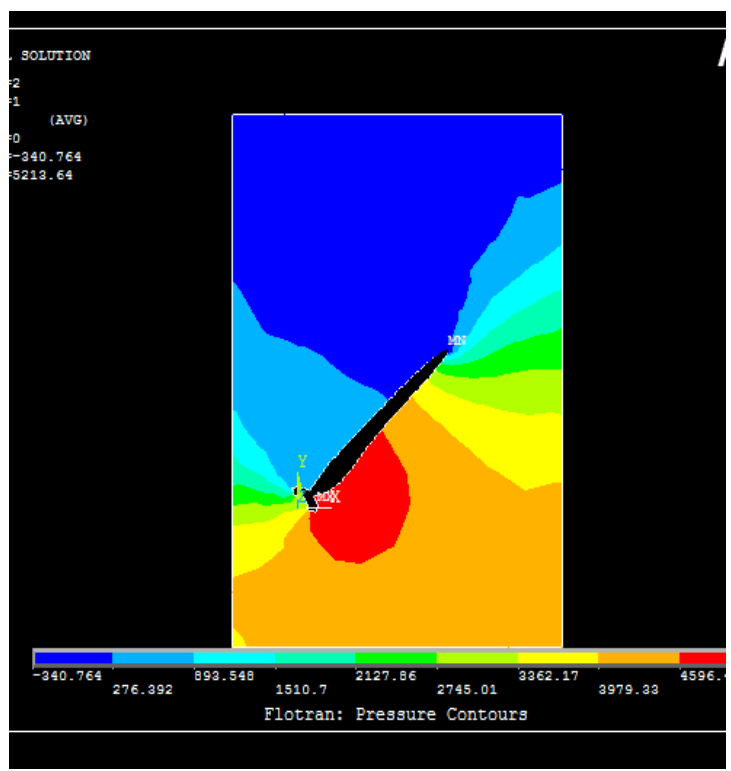

Fig.2: Flotran: Pressure Contours

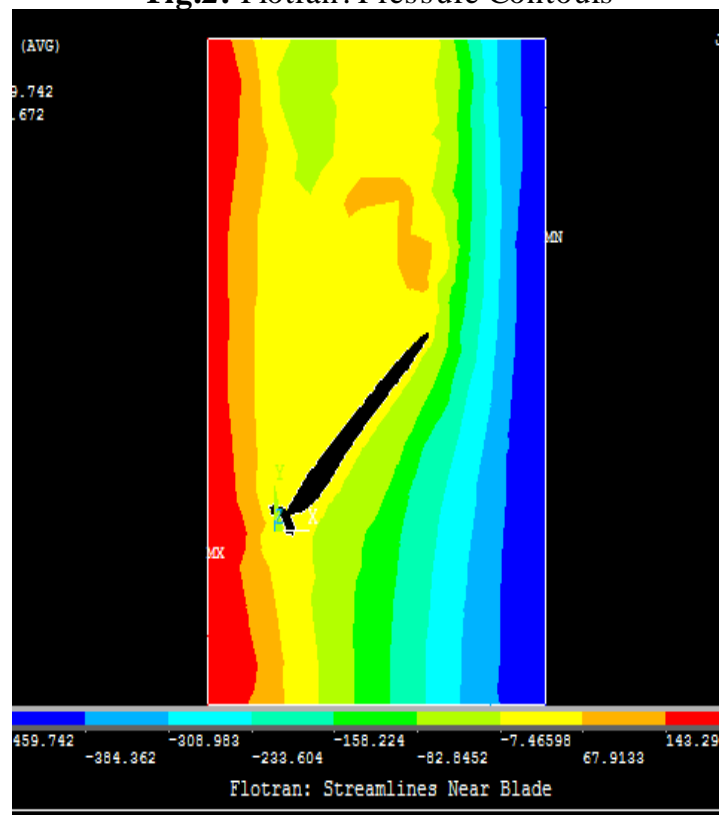

Fig.3: Pressure Contours 
Finally, the peak stress in the final analysis is less than the peak stress in the first analysis. This indicates that considering the effect of the displaced geometry on the flow field made a significant difference.

The mode shape with deformed and un-deformed form is shown in Fig.3.

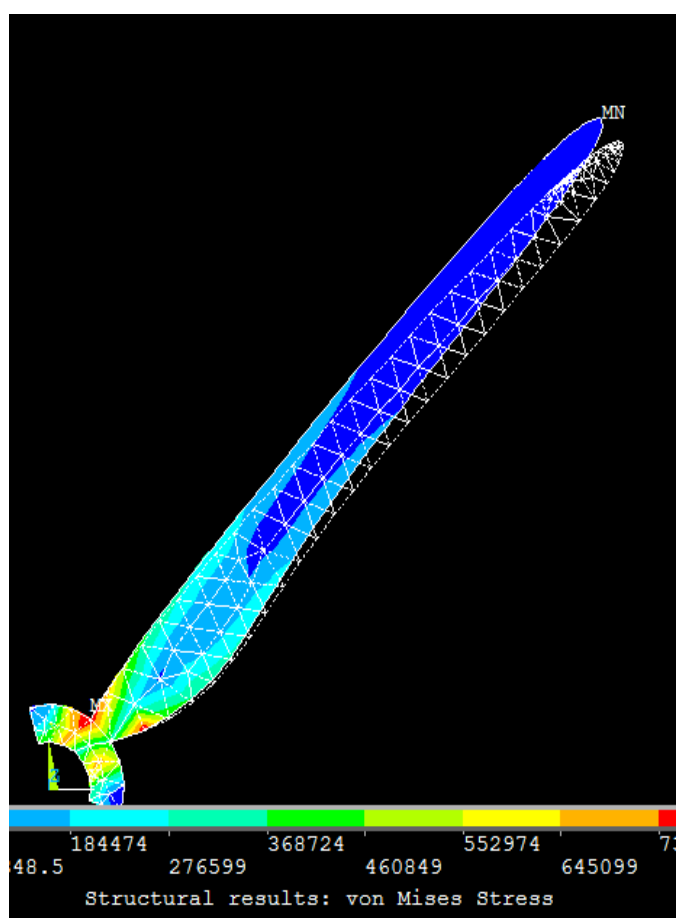

Fig.4: Von Mises stress obtained in the final analysis of blade

\section{MODAL ANALYSIS OF WIND TURBINE BLADE UNDER AIR- FLOW}

In this stage, we can conduct a modal analys is that involves FSI, unsymmetric matrices are generated and hence only an unsymmetric eigensolver can be used. This analysis allows us to determine the natural frequencies mode shapes of a blade subjected to air flow, which are important parameters in the design of a structure for dynamic loading conditions.

Tables 1 and 2 give respectively material's properties of the structure and fluid.

Table - 1 Material properties of the structure

\begin{tabular}{|c|c|c|}
\hline Poisson's ratio & Density $\left[\mathrm{Kg} / \mathrm{m}^{3}\right]$ & Young's modulus $\left[\mathrm{N} / \mathrm{m}^{2}\right]$ \\
\hline $3790 \mathrm{E} 6$ & 0,34 & 2600 \\
\hline
\end{tabular}

Table - 2 Material properties of the flu id

\begin{tabular}{|c|c|}
\hline Speed of sound $\left[\mathbf{m} \boldsymbol{s}^{-1}\right]$ & Density $\left[\mathrm{Kg} / \mathbf{m}^{3}\right]$ \\
\hline 11000 & 0,34 \\
\hline
\end{tabular}

The natural frequencies, obtained from the modal analysis, are presented in Table below:
Table - 3 Natural frequencies of blade under air

flow compared to blade in stagnant fluid [8].

\begin{tabular}{|c|c|c|}
\hline Modes & $\begin{array}{c}\text { Frequency } \\
\text { (stagnant fluid) }\end{array}$ & $\begin{array}{c}\text { Frequency } \\
\text { (flowing fluid) }\end{array}$ \\
\hline 1 & 14,131 & 2,1038 \\
\hline 2 & 15,932 & 11,8366 \\
\hline 3 & 20,114 & 30,6532 \\
\hline 4 & 21,821 & 36,6799 \\
\hline 5 & 28,710 & 58,4824 \\
\hline
\end{tabular}

The performed modal analysis gives estimates of natural frequencies. The results are based on the calculation performed on blade of wind turbine as described in subsection.

\section{PROBABILISTIC APPROACH}

Probabilistic design is an engineering design methodology with the aim to produce highquality products, by systematically studying the effects of variations in the design parameters on product performance. Robust design is a methodology for optimising this quality by making the performance of the product insensitive to variations in the manufacturing, material, operational, and environmental properties [6].

The foundation of probabilistic design involves basing design criteria on reliability targets instead of deterministic criteria. Design parameters such as applied loads, material strength, and operational parameters are researched and/or measured, then statistically defined. A probabilistic analysis model is developed for the entire system and solutions performed to yield failure probability [7].

The focus however will be regarding the beneficial gaining that are associated with probabilistic modeling of vibration of a blade wind turbine compared to a regular deterministic approach using partial safety factors and characteristic values. The deterministic design approach requires great knowledge and a precise description of the blade parameters, which is often a difficult task to determine.

It appears inevitable that the industry, as well as many other industries, will eventually incorporate probabilistic analysis methods to some degree. Probabilistic structural analysis methods, unlike traditional methods, provide a means to quantify the inherent risk of a design and to quantify the sensitivities of design variables.

Monte Carlo simulation (MCS) is a method that is widely used in probabilistic approaches. MCS is to generate set of random samples. The mechanical model is run for each of these samples. Is a relatively easy method for predicting the variation and bias in a system. The results obtained are then used to calculate the estimator of the response [7]. 
Front of the complexity of the problem, we have chosen to consider only the sources of uncertainties related to the material properties and we will be limited to the study of a single blade in air. Table (4) contains the means of random variables used in this study and the distributions laws chosen.

Table - 4 moments and distribution laws of the parameters.

\begin{tabular}{|c|c|c|c|}
\hline Parameters & $\begin{array}{c}\text { Standard } \\
\text { de viation }\end{array}$ & Means & distribution \\
\hline $\begin{array}{c}\text { Young's } \\
\text { modulus [Pa] }\end{array}$ & $1895 \mathrm{E} 4$ & $3790 \mathrm{E} 6$ & Gaussian \\
\hline
\end{tabular}

Table 5 gives the results of determin istic and probabilistic computation for a wind turbine blade.

\begin{tabular}{|c|c|c|c|}
\hline Parameters & $\begin{array}{c}\text { Lower } \\
\text { Boundary }\end{array}$ & $\begin{array}{c}\text { Upper } \\
\text { Boundary }\end{array}$ & distribution \\
\hline $\begin{array}{c}\text { Density of } \\
\text { structure }\end{array}$ & 1300 & 2600 & Uniform \\
\hline $\begin{array}{c}\text { Density of } \\
\text { fluid }\end{array}$ & 0,65 & 1,3 & Uniform \\
\hline
\end{tabular}

Table $-\mathbf{5}$ means of the frequencies for a blade of wind turbine in air.

\begin{tabular}{|c|c|c|}
\hline Frequencies & Deterministic & Probabilistic \\
\hline 1 & 2,1038 & 2,0908354 \\
\hline 2 & 11,837 & 8,2015838 \\
\hline 3 & 30,6532 & 27,0744 \\
\hline 4 & 36,6799 & 41,3867 \\
\hline 5 & 58,4824 & 61,191069 \\
\hline
\end{tabular}

\section{CONCLUSION}

For the fluid-structure interaction problem, we use a load transfer method to illustrate a steadystate problem applied on a blade wind turbine under air flow and demonstrate the use of nonlinear largedeflection structural coupling for a fluid domain. This simulation followed by modal analysis to present influence of the air flow on the natural frequencies of the system.

Taking the uncertainties into account, one probabilistic approach is proposed based on the Monte Carlo simulation. The finagling results show the influence of the uncertainties in the computation of the blade's frequencies. This can give to the engineer another way to design the blade of the wind turbine.

\section{REFERENCES}

[1] K. Takizawa Y. Bazilevs and T.E. Tezduyar, Computational fluid_structure interaction: methods and applications (John Wiley, 2013).

[2] E. Kulunk, Aerodynamics of Wind Turbines. Journal of Fundamental and Advanced Topics in Wind Power, 10.5772/17854, 05 (07), 2011.

[3] H. Cao, Aerodynamics Analysis of Small Horizontal Axis Wind Turbine Blades by Using $2 D$ and $3 D C F D$ Modelling, doctoral diss, University of the Central Lancashire, Preston, 2011.

[4] E. Madenci, and I. Guven. The Finite Element Method and Applications in Engineering Using ANSYS (United States, Springer, 2015).

[5] ANYSYS 13, Coupled-Field Analyses.

[6] A. El Hami, and B. Radi. Uncertainty and Optimization in Structural Mechanics (United Kingdom, Wiley-ISTE, 2013).

[7] B. Dodson, P. C. Hammett and R. Klerx, Probabilistic Design for Optimization and Robustness for Engineers (United Kingdom, Wiley, 2014).

[8] I. Sossey-Alaoui, B. Radi, Modal and probabilistic analysis of wind turbine blade, PARIPEX-Indian Journal of Research, 04(7), 2015, 349-353.

[9] C. Q. Howard and Benjamin S. Cazzolato, Acoustic Analyses using Matlab and ANSYS (Boca Raton, CRC press, 2014)

[10] M. Breuer, M. Münsch, FSI of the Turbulent Flow around a Swiveling Flat Plate Using Large-Eddy Simulation, Fluid-Structure Interaction. Theory, Numerics and Applications, 2008, 31-42.

[11] K. Nordanger, A. Rasheed, K. M. Okstad, A. M. Kvarving, R. Holdahl and T. Kvamsdal, Numerical benchmarking of fluid-structure interaction: An isogeometric finite element approach, Science Direct, 15 (9), 2016, 324-339. 